\title{
INDEX TO VOLUME 78
}

\author{
RESEARCH ANNOUNCEMENTS
}

Allan, N. D. Hecke rings of congruence subgroups, 541.

Allen, H. P. Invariant splittings in nonassociative algebras: A Hopf approach, 245.

Amdursky, V. The existence of solutions to classical variational problems without assuming convexity, 394.

Anderson, D. R. Generalized product theorems for torsion invariants with applications to flat bundles, 465 .

Anderson, D. W. A generalization of the Eilenberg-Moore spectral sequence, 784.

Andersson, K. G. Propagation of analyticity for solutions of differential equations of principal type, 479 .

Andre, Michel. Nonnoetherian complete intersections, 724.

Arkeryd, Leif. An existence theorem for a modified space-inhomogeneous, nonlinear Boltzmann equation, 610.

Aron, R. M. Holomorphic functions on balanced subsets of a Banach space, 624.

Arveson, William. Lattices of invariant subspaces, 515.

Auslander, Louis and Brezin, Jonathan. Invariant subspace theory for three-dimensional nilmanifolds, 255.

Baernstein, Albert II. Proof of Edrei's spread conjecture, 277.

Baouendi, M. S. and Goulaouic, C. Nonanalytic-hypoellipticity for some degenerate elliptic operators, 483.

Batt, Jürgen. Strongly additive transformations and integral representations with measures of nonlinear operators, 474.

Baumslag, Gilbert. On finitely presented metabelian groups, 279.

Beals, Richard. Mixed boundary value problems for nonstrict hyperbolic equations, 520.

Berger, M. S. On Hermitian structures of prescribed nonpositive Hermitian scalar curvature, 734.

Berger, M. S. and Schechter, Martin. On the solvability of semilinear operator equations and elliptic boundary value problems, 741 .

Birman, J. S. and Hilden, H. M. Isotopies of homeomorphisms of Riemann surfaces and a theorem about Artin's braid group, 1002.

Bott, R. and Haefliger, A. On characteristic classes of $\Gamma$-foliations, 1039.

Bourgin, D. G. Set valued transformations, 597.

Brezin, Jonathan. See Auslander, Louis.

Brooks, J. K. Weak compactness in the space of vector measures, 284.

Brooks, J. K. and Lewis, P. W. Operators on functions spaces, 697.

Brosowski, Bruno and Deutsch, Frank. Some new continuity concepts for metric projections, 974.

Browder, F. E. Generalizations of accretivity for nonlinear mappings of Banach spaces, 93. .Normal solvability and $\phi$-accretive mappings of Banach spaces, 186.

Brown, Marianne. Constructing isotopies in noncompact 3-manifolds, 461.

Campbell, D. M. Majorization-subordination theorems for locally univalent functions, 535.

Cappell, S. E. and Shaneson, J. L. Submanifolds, group actions and knots. I, 1045.

. Submanifolds, group actions and knots. II, 1049.

Cellina, Arrigo. On the nonexistence of solutions of differential equations in nonreflexive spaces, 1069.

Chang, B. C. Which abelian groups can be fundamental groups of regions in Euclidean spaces?, 470 .

Chang, Theodore and Skjelbred, Tor. Group actions on Poincaré duality spaces, 1024.

Chapman, T. A. Characterizing shapes of compacta, 847.

Chen, B.-Y. Surfaces with parallel mean curvature vector, 709.

Chen, B.-Y. and Ludden, G. D. Rigidity theorems for surfaces in euclidean space, 72.

Chernoff, P. R. Nonassociative addition of unbounded operators and a problem of Brezis and Pazy, 562.

Chvátal, Václav and Harary, Frank. Generalized Ramsey theory for graphs, 423.

Clark, D. N. Extending Fourier transforms into Sz.-Nagy-Foiaş spaces, 65.

Coffee, J. P. Filtered and associated graded rings, 584.

Cohen, J. M. A note on Poincaré 2-complexes, 763.

Csordas, G. L. and Reiter, H. B. Some results on separating function algebras, 578. 
Dennis, R. K. and Stein, M. R. A new exact sequence for $K_{2}$ and some consequences for rings of integers, 600 .

DeMar, R. F. Multiple expansions in series of Boas and Buck polynomials, 550.

Deutsch, Frank. See Brosowski, Bruno.

Dickson, L. J. Estimates for the Szegö and Poisson kernels of sufficiently rounded tube domains, 844.

Dlab, Vlastimil and Ringel, C. M. Balanced local rings with commutative residue fields, 771 .

Duvall, P. F., Jr. and Husch, L. S. Taming irregular sets of homeomorphisms, 77.

Dyer, J. A., Pedersen, E. A. and Porcelli, P. An equivalent formulation of the invariant subspace conjecture, 1020.

Edelstein, Michael. The construction of an asymptotic center with a fixed-point property, 206.

Eifler, L. Q. Nonseparating function algebras, 604.

Eisenbud, David and Evans, E. G., Jr. Basic elements: Theorems from algebraic K-theory, 546.

Elliott, R. J. and Kalton, N. J. Values in differential games, 427.

Evans, E. G., Jr. See Eisenbud, David.

Ewing, John. On the type of associative H-spaces, 35.

Faith, Carl. Galois subrings of Ore domains are Ore domains, 1077.

FitzGerald, C. H. Exponentiation of certain quadratic inequalities for Schlicht functions, 209.

Fitzpatrick, P. M. A-proper mappings and their uniform limits, 806.

Ford, Charles. Pure, normal maximal subfields for division algebras in the Schur subgroup, 810.

Freese, Ralph. Varieties generated by modular lattices of width four, 447.

Garland, Howard. p-adic curvature and a conjecture of Serre, 259.

Gelbart, S. S. Harmonics on Stiefel manifolds and generalized Hankel transforms, 451.

Geoghegan, Ross and Summerhill, R. R. Infinite-dimensional methods in finite-dimensional geometric topology, 1009.

Gersten, S. M. On the spectrum of algebraic K-theory, 216.

Gerstenhaber, Murray. The fundamental form of a finite purely inseparable field extension, 717.

Gilmer, Robert. On Prüfer rings, 223.

Gilbert, R. P. and Hile, G. N. Generalized hyperanalytic function theory, 998.

Goguen, J. A. Minimal realization of machines in closed categories, 777.

Goldstein, Charles. The $\mathscr{S}$-matrix associated with nonselfadjoint differential operators, 411.

Goldstein, L. J. Relative imaginary quadratic fields of low class number, 80.

Gordon, W. B. Physical variational principles which satisfy the Palais-Smale condition, 712.

Gorostiza, L. G. A central limit theorem for a class of d-dimensional random motions with constant speed, 575.

Gossez, J.-P. Boundary value problems for quasilinear elliptic equations with rapidly increasing coefficients, 753.

Goulaouic, C. See Baouendi, M. S.

Greene, R. E. and Wu, H. Curvature and complex analysis. II, 866.

Griess, R. L., Jr. Schur multipliers of the known finite simple groups, 68.

Grünbaum, F. A. An inverse problem for Gaussian processes, 615. . On the existence of a "Wave Operator" for the Boltzmann equation, 759.

Guillemin, Victor. A remark on strong pseudoconvexity for elliptic operators, 432. Remarks on some results of Gelfand and Fuks, 539.

Haefliger, A. See Bott, R.

Hajnal, A. and Juhảsz, I. Two consistency results in topology, 711.

Hallam, T. G. A terminal comparison principle for differential inequalities, 230.

Hansell, R. W. On the nonseparable theory of Borel and Souslin sets, 236.

Harary, Frank. See Chvátal, Václav.

Harper, J. R. Homotopy groups of finite H-spaces, 532.

Harte, Robin. The spectral mapping theorem in several variables, 871.

Hatcher, A. E. The second obstruction for pseudo-isotopies, 1005.

Helton, J. W. Jordan operators in infinite dimensions and Sturm Liouville conjugate point theory, 57.

Herrero, D. A. and Salinas, Norberto. Operators with disconnected spectra are dense, 525.

Hilden, H. M. See Birman, J. S.

Hile, G. N. See Gilbert, R. P.

Hilton, Peter, Mislin, Guido, and Roitberg, Joseph. Topological localization and nilpotent groups, 1060.

Hoffman, D. A. Surfaces in constant curvature manifolds with parallel mean curvature vector field, 247 .

Holzsager, Richard. Reimannian manifolds of finite order, 200.

Howland, J. S. Perturbation of embedded eigenvalues, 280. 
Huckleberry, A. T. and Nirenberg, Ricardo. On a class of complex spaces intermediate to Stein and compact, 852.

Husch, L. S. See Duvall, P. F., Jr.

Iarrobino, A. Punctual Hilbert schemes, 819.

Iitaka, Shigeru. On algebraic varieties whose universal covering manifolds are complex affine 3-spaces, 737.

Jaworowski, J. W. Extensions of maps in spaces with periodic homeomorphisms, 527.

Jensen, C. U. On $\operatorname{Ext}_{R}^{1}(A, R)$ for torsion-free $A, 831$.

Johnson, Jerry. Lipschitz function spaces for arbitrary metrics, 702.

Johnson, Kenneth and Wallach, N. R. Composition series and intertwining operators for the spherical principal series, 1053.

Jones, Lowell. The converse to the fixed point theorem of P. A. Smith, 234.

. Three characteristic classes measuring the obstruction to PL local unknotedness, 979.

Juberg, R. K. Finite Hilbert transforms in $L^{p}, 435$.

Juhasz, I. See Hajnal, A.

Kahn, D. S. and Priddy, S. B. Applications of the transfer to stable homotopy theory, 981.

Kalton, N. J. See Elliott, R. J.

Karel, M. L. Fourier coefficients of certain Eisenstein series, 828.

Karlin, Samuel. On a class of best nonlinear approximation problems, 43.

Kazdan, J. L. and Warner, F. W. Curvature functions for 2-manifolds with negative Euler characteristic, 570.

Kim, Ohoe. A representation of a positive linear mapping, 568.

Kinderlehrer, David. How a minimal surface leaves an obstacle, 969.

Knebusch, Manfred, Rosenberg, Alex and Ware, Roger. Signatures on semilocal rings, 62.

Knopfmacher, John. Finite modules and algebras over Dedekind domains and analytic number theory, 193.

Kra, I. and Maskit, B. Involutions on Kleinian groups, 801.

Kronk, H. V. and Mitchem, John. The entire chromatic number of a normal graph is at most seven, 799.

Kueker, D. W. Löwenheim-Skolem and interpolation theorems in infinitary languages, 211.

Lambek, J. Localization and completion, 582.

Laplaza, M. L. Coherence for categories with associativity, commutativity and distributivity, 220.

Laudenbach, F. On the 2-sphere in a 3-manifold, 792.

Lazar, A. J. and Taylor, D. C. Double centralizers of Pedersen's ideal of a C*-algebra, 992.

Lazarov, Connor and Wasserman, Arthur. Cobordism of U(n)-actions, 1035.

Levitt, Norman and Morgan, J. W. Transversality structures and p.l. structures on spherical fibrations, 1064.

Lewis, D. C. A generalized Möbius inversion formula, 558.

Lewis, P. W. See Brooks, J. K.

Ludden, G. D. See Chen, B.-Y.

McAuley, L. F. A topological Reeb-Milnor-Rosen theorem and characterizations of manifolds, 82.

Marcus, Moshe. A radial averaging transformation, capacity and conformal radius, 456

Marcus, Moshe and Mizel, V. J. Functional composition on Sobolev spaces, 38

Margolis, H. R. On the realizability of modules over the Steenrod algebra, 564.

Maserick, P. H. BV-functions on commutative semigroups, 202.

Maskit, B. See Kra, I.

Milgram, R. J. On the Haefliger knot groups, 861.

Mislin, Guido. See Hilton; Peter.

Mitchem, John. See Kronk, H. V.

Mizel, V. J. See Marcus, Moshe.

Montague, J. S. and Plemmons, R. J. Convex matrix equations, 965.

Moreno, C. J. Prime number theorems for the coefficients of modular forms, 796.

Morgan, J. W. See Levitt, Norman.

Morrow, J. A. Compactifications of $C^{2}, 813$.

Neuberger, J. W. Quasi-analyticity and semigroups of bounded linear transformations, 85.

Neville, C. W. Invariant subspaces of Hardy classes on infinitely connected plane domains, 857.

Newman, C. M. The inner product of path space measures corresponding to random processes with independent increments, 268.

Nirenberg, Ricardo. See Huckleberry, A. T.

Osborn, Howard. PL sheaves and their characteristic classes, 787.

Palmer, T. W. Hermitian Banach *-algebras, 522.

Parry, William and Walters, Peter. Endomorphisms of a Lebesgue space, 272. 
Patil, D. J. Representation of $H^{p}$-functions, 617.

Pedersen, E. A. See Dyer, J. A.

Petryshyn, W. V. A new fixed point theorem and its application, 225.

Petryshyn, W. V. and Williamson, T. E., Jr. A necessary and sufficient condition for the convergence of a sequence of iterates for quasi-nonexpansive mappings, 1027.

Pettey, D. H. Mapping; into 2-dimensional spaces, 53.

Pincus, David. Independence of the prime ideal theorem from the Hahn Banach theorem, 766.

Pinsky, M. A. Limit theorems for continuous state branching processes with immigration, 242.

Pittenger, A. O. and Shih, C. T. Coterminal families and the strong Markov property, 439.

Plemmons, R. J. See Montague, J. S.

Porcelli, P. See Dyer, J. A.

Priddy, S. B. See Kahn, D. S.

Quinn, Frank. Surgery on Poincaré and normal spaces, 262.

Range, R. M. and Siu, Y.-T. Uniform estimates for the $\partial$-equation on intersections of strictly pseudoconvex domains, 721.

Rao, M. L. R. Azumaya, semisimple and ideal algebras, 588.

Reed, M. C. and Simon, Barry. A spectral theorem for tensor products of unbounded operators, 730.

Reiter, H. B. See Csordas, G. L.

Rieffel, M. A. Induced representations of $C^{*}$-algebras, 606.

Ringel, C. M. See Dlab, Vlastimil.

Roberts, Joel. Singularity subschemes and generic projections, 706.

Roggenkamp, K. W. Classification of the completely primary totally ramified orders with a finite number of nonisomorphic indecomposable lattices, 399.

Roitberg, Joseph. See Hilton, Peter.

Rosenberg, Alex. See Knebusch, Manfred.

Rothaus, O. S. Study of the permanent conjecture and some generalizations, 749.

Rubin, R. A. Absolutely torsion-free rings, 854.

Ruelle David. Statistical mechanics on a compact set with $Z^{v}$ action satisfying expansiveness and specification, 988.

Salinas, Norberto. See Herrero, D. A.

Sally, P. J., Jr. and Warner, Garth. Fourier inversion for semisimple Lie groups of real rank one, 251.

Schechter, Martin. See Berger, M. S.

Scheinberg, Stephen. The spectrum of an automorphism, 621.

Schori, R. and West J. E. $2^{1}$ is homeomorphic to the Hilbert cube, 402.

Schrader, Keith. A generalization of the Helly selection theorem, 415.

Shaneson, J. L. See Cappell, Sylvain E.

Shih, C. T. See Pittenger, A. O.

Shub, Michael. Structurally stable diffeomorphisms are dense, 817.

Simon, Barry. See Reed, M. C.

Siu, Y.-T. See Range, R. M.

A Thullen type extension theorem for positive holomorphic vector bundles, 775 .

Skjelbred, Tor. See Chang, Theodore.

Sloan, Alan. A relativistic polaron without cutoffs in two space dimensions, 407.

Smith, R. T. Harmonic mappings of spheres, 593.

Sonn, Jack. On the embedding problem for nonsolvable Galois groups of algebraic number fields: Reduction theorems, 553.

Stein, M. R. See Dennis, R. K.

Summerhill, R. R. See Geoghegan, Ross.

Sunley, J. E. On the class numbers of totally imaginary quadratic extensions of totally real fields, 74.

Taylor, D. C. See Lazar, A. J.

Thurston, William. Noncobordant foliations of $S^{3}, 511$.

Tong, Y. L. L. deRham's integrals and Lefschetz fixed point formula for d" cohomology, 420.

Torchinsky, Alberto. Singular integrals in the spaces $\Lambda(B, X), 1015$.

Uhlenbeck, K. Eigenfunctions of Laplace operators, 1073.

Veech, W. A. A converse to Gauss' theorem, 444.

Wallach, N. R. See Johnson, Kenneth.

. Three new examples of compact manifold admitting Riemannian structures of positive curvature, 55.

Walsh, J. J. Fiber preserving cellular decompositions, 746.

Walsh, T. On the summation of conjugate Fourier integrals of functions of several variables, 840.

Walter, M. E. The dual group of the Fourier-Stieltjes algebra, 824. 
Walters, Peter. See Parry, William.

Wang, Kai. Differential actions on $2 n$-spheres, 971.

Ware, Roger. See Knebusch, Manfred.

Warfield, R. B., Jr. Classification theorems for p-groups and modules over a discrete valuation ring, 88.

Warner, F. W. See Kazdan, J. L.

Warner, Garth. See Sally, P. J., Jr.

Wasserman, Arthur. See Lazarov, Connor.

Watson, Bill. The first Betti numbers of certain locally trivial fibre spaces, 392.

West, J. E. See Schori, R.

Whitten, Wilbur. Surgically transforming links into noninvertible knots, 99.

Williamson, T. E., Jr. See Petryshyn, W. V.

Wright, E. M. The number of unlabelled graphs with many nodes and edges, 1032.

$\mathrm{Wu}, \mathrm{H}$. See Greene, R. E.

Yen, C.-L. The range of m-dissipative sets, 197.

Yood, Bertram. Incomplete normed algebras, 50.

Zachmanoglou, E. C. Foliations and the propagation of zeroes of solutions of partial differential equations, 835.

\section{BOOK REVIEWS}

Arnold, V. Review of "Celestial mechanics. I, II" by Shlomo Sternberg, 962.

Corwin, Lawrence. Review of "Algebraic number theory" by Serge Lang, 690 .

Figà-Talamanca, Alessandro. Review of "Abstract harmonic analysis. I, II" by E. Hewitt and K. A. Ross, 172.

Griffiths, P. Review of "Hyperbolic manifolds and holomorphic mappings" by Shoshichi Kobayashi, 487.

Keesling, James. Review of "Dimension theory" by Keiô Nagami, 953.

Knight, F. B. Review of "Diffusion processes and their sample paths" by Kiyosi Ito and Henry P. McKean, Jr., 956.

Mycielski, Jan. Review of "Perceptrons, An introduction to computational geometry" by Marvin Minsky and Seymour Papert, 12.

Ragozin, D. L. Review of "Methods of Hilbert spaces" by Krzysztof Maurin; "General eigenfunction expansions and unitary representations of topological groups" by Krzysztof Maurin, 15.

Rheinboldt, W. C. Review of "Modern applied algebras" by Garrett Birkhoff and Thomas C. Bartee, 383 .

Stein, P. R. Review of "Combinatorial identities" by John Riordan, 490.

Walsh, Bertram. Review of "Introduction to the theory of partially ordered spaces" by B. Z. Vulikh, 178.

Waterhouse, W. C. Review of "Lebesgue's theory of integration" by Thomas Hawkins; "A history of vector analysis" by Michael J. Crowe; "The development of the foundations of mathematical analysis from Euler to Riemann" by I. Grattan-Guinness; "Die Genesis des abstrakten Gruppenbegriffes" by Hans Wussing, 385 .

Woyczynski, W. A. Review of "Best approximation in normed linear spaces by elements of linear subspaces" by Ivan Singer, 685 .

\section{REPORTS OF MEETINGS AND MISCELLANEOUS ARTICLES}

Bateman, P. T. Reports of Meetings of The American Mathematical Society: The November meeting in Milwaukee, 184; The April meeting in St. Louis, 508.

Chen, B.-Y. and Ludden, G. D. Errata to "Rigidity theorems for surfaces in euclidean space", 628.

Council and Board of Trustees-1971, 104.

Edelstein, Michael. Errata to: "The construction of an asymptotic center with a fixed-point property", 1087.

Garland, H. Errata to: "p-adic curvature and a conjecture of Serre", 1087.

Gottschalk, Walter. See Pitcher, Everett.

Gottschalk, Walter. Reports of Meetings of The American Mathematical Society: The October meeting in Cambridge, 182; The March meeting in New York, 507.

Hagopian, C. L. Addendum to: "A fixed point theorem for plane continua", 289.

Harrold, O. G. Reports of Meetings of the American Mathematical Society: The November 
meeting in Auburn, Alabama, 183.

Ludden, G. D. See Chen, B.-Y.

Martin, W. T. Report of the Treasurer-1971, 694.

Parry, William and Walters, Peter. Erratum to "Endomorphisms of a Lebesgue space", 628.

Pitcher, Everett. 1971 Election Results, 288.

Erratum to: "The Bylaws of the American Mathematical Society", 290.

Pitcher, Everett and Ross, K. A. Reports of Meetings of The American Mathematical Society: The annual meeting in Las Vegas, 497.

Pitcher, Everett and Gottschalk, Walter. Reports of Meetings of The American Mathematical Society: The Summer meeting in University Park, 21.

Ross, Kenneth. Reports of Meetings of The American Mathematical Society: The April meeting in Berkeley, 510; The June meeting in Seattle, 964.

Statement of Ownership, Management \& Circulation, 1088.

Walters, Peter. See Parry, William.

\section{INVITED ADDRESSES}

Ax, J. B., 507.

Baumslag, Gilbert, 509.

Boardman, J. M., 21.

Browder, F. E., 21.

Buchsbaum, D. A., 507.

Cantrell, J. C., 183.

Devinatz, Allen, 508.

Dyson, F. J. Missed opportunities, 635.

Griffiths, P. A. Holomorphic mappings: Survey of some results and discussion of open problems, 374.

Hofmann, K. H. Representations of algebras by continuous sections, 291.

$\mathrm{Kohn}, \mathrm{J}$. J. Integration of complex vector fields, 1.

Lawson, Blain, 497.

McArthur, C. W., 183. Developments in Schauder basis theory, 877.

Marcus, Lawrence, 509.

Mazur, Barry, 182.

Frobenius and the Hodge filtration, 653.

Narasimhan, Raghavan, 184.

Neuberger, J., 183. Quasi-analyticity and semigroups, 909.

Palais, R. S., 497.

Petrie, Ted. Smooth $S^{1}$ actions on homotopy complex projective spaces and related topics, 105.

Pugh, C. C., 497.

Richardson, Roger, 510

Robbin, J. W., 21. Topological conjugacy and structural stability for discrete dynamical systems, 923.

Rosenberg, Alex, 507.

Rossi, Hugo, 497.

Rota, G.-C., 507.

Rudin, M.-E., 184 .

Sacks, G. E., 182.

The differential closure of a differential field, 629.

Schmidt, W. M., 508.

Schwartz, J. T., 497.

Shah, S. M. Analytic functions with univalent derivatives and entire functions of exponential type, 154.

Solovay, R. M., 497.

Taylor, J. L., 510.

Tierney, Miles, 497.

Weiss, Benjamin, 21.

The isomorphism problem in ergodic theory, 668.

Williams, R. F., 497. 